\title{
Crise, feminismos e comunicação
}

Em outubro de 2019, a equipe editorial da Revista Eco-Pós discutia as temáticas para os dossiês do próximo ano. Maria Bogado sugeriu o tema de perspectivas e teorias feministas sobre a comunicação. Naquele momento, o lançamento do livro Explosão Feminista, organizado por Heloísa Buarque de Hollanda, indicava a relevância de nosso campo para a compreensão do tema. Liv Sovik, co-editora da revista, sugeriu o nome de Lígia Lana que, entre 2013 e 2015, havia sido professora da disciplina Mídia e representações do feminino na Escola de Comunicação da UFRJ. Com um primeiro esboço em mãos, Maria e Lígia começaram a discutir o tema com Liv Sovik, que se integrou ao coletivo - assim nós nos intitulamos - responsável por este dossiê.

Em dezembro do ano passado, casos de Covid-19 já haviam sido notificados na China, e a Organização Mundial de Saúde indicava a possível epidemia do novo coronavírus. Entretanto, a proporção da pandemia não era imaginada naquele momento. 0 Call For Papers indicava que a ascensão da extrema direita ameaçava as bases da democracia representativa, e o dossiê buscava contribuições que permitissem avaliar as críticas e a mobilização dos feminismos contra as violações de direitos e os ataques aos agentes e às instituições democráticas. Avistava-se, ainda, investigar como os diferentes feminismos formam redes informais de resistência com múltiplas frentes, seja nos ativismos, nas elaborações estéticas, nas lutas partidárias e, sobretudo, nas proposições epistemológicas desafiadoras. Quase ninguém imaginava que a gravidade da pandemia Covid-19 tornaria ainda mais urgente a reflexão sobre as crises acentuadas pelo neoliberalismo que os feminismos já estavam enfrentando.

Na história da Revista Eco-Pós, este é o segundo dossiê dedicado ao tema de problemas de gênero. Há dez anos, a edição Comunicação, Gênero e Cultura das 
Minorias trouxe artigos a respeito das questões de gêneros e minorias. A Revista Eco-Pós apresenta agora esta nova chance de debater questões de gênero, destacando desta vez a palavra feminismos para a compreensão do assunto.

Antes de apresentar os trabalhos selecionados, é preciso ressaltar que o CFP recebeu um número recorde de artigos: foram 47 submissões. Ser um coletivo tornou mais leve o intenso trabalho de organizar o material, enviar para avaliação cega e discutir os pareceres. 0 auxílio do editor Lucas Murari foi fundamental para a atividade. Agradecemos também a todas e todos os pareceristas que contribuíram com a avaliação dos trabalhos. Diante da riqueza do material recebido, não foi possível acolher todos os textos nesta edição - teremos, portanto, um segundo número no começo do ano que vem.

Os textos incluídos neste número, reflexo das submissões, mostram uma preocupação com a profunda violência das relações de gênero e raciais e também com o que sustenta feministas apesar das dificuldades e hostilidade que enfrentam.

"Vênus em dois atos", da escritora e professora Saidiya Hartman, é um texto já consagrado, mas inédito em português, que enfrenta o problema de como reconhecer e fazer reparação às vítimas de violência racista no passado escravagista. Como registrar o valor das vidas de pessoas que somente aparecem no arquivo (livros de tribunais, de contabilidade do tráfico negreiro, diários de seus torturadores) do sistema que as condenou à morte? Entre a recusa da romantização e os traços escritos deixados por brancos que apelidavam de "Vênus" as mulheres escravizadas que mais gostavam de estuprar, Hartman escreve no limite do indizível e do desconhecido. De interesse geral e também particular para a área de Comunicação, pela sua reflexão sobre a narração da história do presente, a tradução cuidadosa foi gentilmente cedida por Fernanda Silva e Sousa e Marcelo R. S. Ribeiro em antecipação de sua inclusão em uma antologia de black studies no ano que vem.

Em "Musas insubmissas: Estudo de "Inês" (1974), um filme de coletivo sobre uma presa política brasileira", Patrícia Furtado Mendes Machado e Thais Blank contam a história de um curta-metragem que é, de uma vez, filme de arte e ação de 
solidariedade e reivindicação da vida de uma presa política brasileira. Inês (1974), que nunca foi mostrado no Brasil, foi realizado por um coletivo feminista francesa (Les Insoumuses) em prol da libertação da prisão de Inês Etienne Romeu. A análise retoma a história da personagem do título, resgata o processo de fabricação da obra e, como "Vênus em dois atos", discute a vontade e as dificuldades de narrar as vidas de pessoas que passam por porões.

Fernanda Carrera e Daniel Meirinho apresentam, em " Mulheres Negras nas Artes Visuais: modos de resistência às imagens coloniais de controle", outra visão do problema da estética da resistência à desumanização, desta vez contemplando os padrões contemporâneos de resistência a "imagens controladoras", termo de Patricia Hill Collins, nas artes visuais. São apontados três modos de resistência a essas imagens: enfrentamentos à submissão, ressignificação das ausências e reivindicação da autonomia sexual, contribuindo para o olhar epistemológico sobre estas manifestações artísticas e seus impactos comunicacionais.

"Estraga-prazeres feministas e outras sujeitas voluntariosas", texto de Sara Ahmed, traduzido de maneira precisa por Luiza Quental, propõe a análise da figura "estraga-prazeres feminista", enquadrando o pensamento feminista como uma crítica da felicidade. A obstinação feminista é entendida como o terreno incerto para uma política coletiva de tradução de emoções individuais, dor ou raiva diante das injustiças. Além disso, a figura feminista voluntariosa pode ajudar a compreender as formas pelas quais, nos espaços feministas, as mulheres negras foram reduzidas à sua raiva e designadas como causadoras das divisões produzidas pelo racismo.

Ana Carolina Escosteguy recupera, em "Comunicação e gênero no Brasil: discutindo a relação", as vinculações entre os estudos de comunicação e questões de gênero no Brasil. Com um olhar histórico, destacam-se alguns momentos-chave: na arrancada inaugural (1970/1980), sobressai o uso sistemático da categoria mulher; no segundo impulso (1990), embora o termo gênero seja acionado, funciona mais como etiqueta; no terceiro (2000-2015), é a crítica ao pós-feminismo que desponta, evidenciando a primeira convergência entre Sul e Norte. Por fim, o último impulso recém se desenha a partir da primavera feminista e o horizonte aberto pela explosão 
dos feminismos, impulsionado pelas novas mídias digitais, bem como a ascensão de uma extrema-direita que potencializa e promove um discurso antifeminista.

Tatiane Leal, em "O sentimento que nos faz irmãs: construções discursivas da sororidade", analisa as definições de sororidade presentes nos links mais compartilhados sobre o tema em sites de redes sociais. Dois eixos são explorados: a associação do conceito ao campo semântico dos sentimentos, especialmente à empatia; e as diferentes origens atribuídas à sororidade, vista ora como parte de uma essência feminina, ora como resultado de uma postura ético-política desenvolvida a partir da sociabilidade entre mulheres. A investigação revela o nexo entre sentimentos, moralidade e política em processos comunicacionais contemporâneos.

Em "Mulher, Negra e Repórter: atravessamentos entre gênero, raça, subjetividade e telejornalismo na trajetória de Glória Maria”, Valéria Maria Villas Boas analisa a trajetória da jornalista Glória Maria, buscando compreender os atravessamentos entre subjetividade, jornalismo, raça e gênero. A pesquisa mostra que a noção de objetividade jornalística opera como modo de regulação de expressões subjetivas a partir de uma articulação de sentido entre corpos convencionados como sóbrios, discretos e os valores de credibilidade e autoridade a eles confiados.

Em "Lugar de mulher é no YouTube: o feminismo popular de influenciadoras digitais socialmente engajadas", Simone Evangelista discute a presença dos feminismos em plataformas digitais a partir da atuação de influenciadoras digitais. Contextualiza o problema na discussão de perspectivas teóricas sobre a própria viabilidade de um discurso feminismo nesse meio. Três canais no YouTube são examinados: Afros e Afins, Jout Prazer e Nunca te Pedi Nada. O trabalho indica a necessidade de repensar a noção de feminismo popular a partir de nuances mais diversas e complexas.

A violência obstétrica é o tema de Veronica Aparecida Ribeiro Haacke e Fábio Malini, em "'Você fez, agora aguenta': Análise das narrativas de violência obstétrica no Facebook". Analisam comentários presentes em páginas do Facebook e investigam as redes como espaço de discussão, seja como maneira de agrupar 
mulheres vítimas - a partir do compartilhamento de seus relatos e experiências - ou como forma de conscientização a partir da divulgação de informações.

Nós tivemos ainda a alegria de entrevistar Heloísa Buarque de Hollanda, que, com sua lucidez e honestidade, traz inspirações para pensar a ideia dos "feminismos em pleno voo". As palavras de Heloísa trazem inquietações, mas também alento e entusiasmo, uma vez que sugere as lutas feministas hoje como espaços de multiplicidade e novos protagonismos, em busca da transformação social.

O portfólio apresenta o trabalho do artista múltiplo Elton Panamby, cujo meio principal é o próprio corpo. Figura presente na cena artística jovem e em diversos cantos da Internet, tem uma trajetória artística e intelectual em múltiplas parcerias e no esforço de criação de territórios de encontro e troca de saberes fora das instituições convencionais de arte e ensino. Elton marcou de modo crítico o pensamento queer e transfeminista na década de 2010 e impactou muitxs jovens em seus processos de arte e vida. Conhecer seu trabalho abriu o espírito dos dois terços do coletivo editorial que não o conhecia. Agradecemos a Elton pela cordialidade de permitir a veiculação de uma imagem de "Sob o Sol das Cabras" como nossa imagem de capa.

Por fim, Maria Santos Castanho Caú resenha o livro "Mulheres atrás das câmeras", publicado em 2019 por Luiza Lusvarghi e Camila Vieira da Silva. O trabalho repercute o apagamento do cinema brasileiro feito por mulheres, indicando a importância da obra no contexto da construção de nova historiografia do cinema brasileiro.

Como editoras, terminamos nossa atividade com a certeza que pode ser viável uma revolução dos femininos e dos feminismos, com união e gentileza, sem perder de vista as lutas.

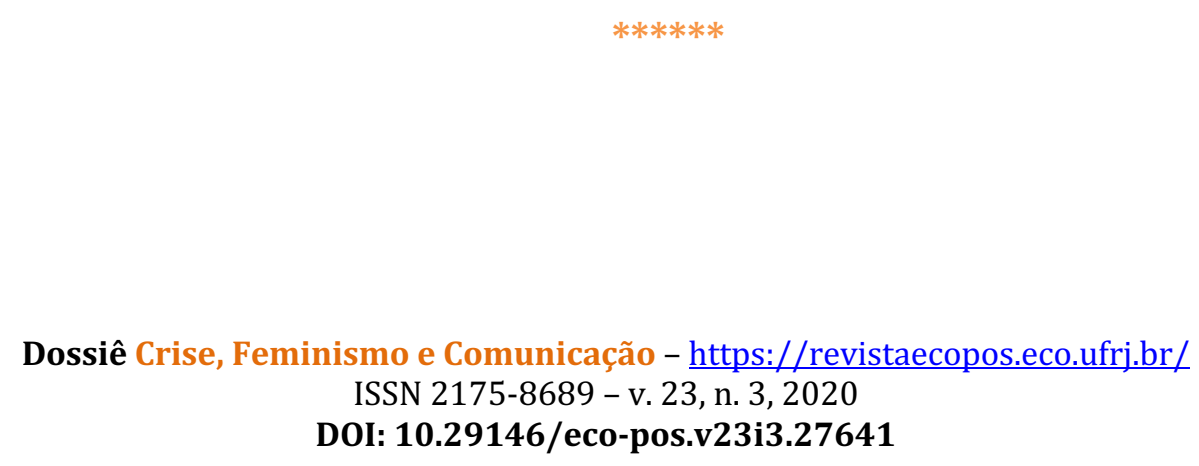


O terceiro e último número de 2020 da Revista publica oito artigos de temática "Livre" relacionados ao campo da Comunicação e Cultura. A Seção Perspectiva tem início com o artigo de Francisco Rüdiger, "A comunicação como aventura solipsística: sobre a "nova teoria" de Ciro Marcondes Filho", reflexão que busca examinar os fundamentos teóricos dos estudos feitos por Ciro Marcondes Filho ao longo de décadas de atividade intelectual. 0 professor e pesquisador foi um dos mais ativos na área da Comunicação, e faleceu no último dia 08 de Novembro. $\mathrm{A}$ primeira versão do artigo de Rüdiger foi enviada para a Eco-Pós no início de 2020. Nesse hiato entre a submissão, o aceite, as modificações solicitadas e a publicação, o texto foi enviado para o próprio Ciro Marcondes Filho, que leu com atenção e nos enviou um artigo resposta em torno das problematizações apresentadas por Rüdiger. A equipe editorial do periódico, em homenagem ao professor recémfalecido, decidiu também publicar neste mesmo número sua réplica: "Reabilitando o Positivismo: Francisco Rüdiger "critica" a Nova Teria da Comunicação. Mas não impunemente", fomentando o debate de ideias entre os dois autores.

Dando continuidade a esta seção, Eduardo Harry Luersen é autor de “O corpo suturado: Interfaces sonoras e a construção das condições de audibilidade dos jogos digitais", investigação que se concentra no conceito de interface e nas discussões de arqueologia das mídias para investigar o design sonoro dos jogos digitais em primeira e terceira pessoa. "You Better Work: 0 trabalho nos Estudos de Comunicação e Música no Brasil”, de Adriana da Rosa Amaral e Rafael do Nascimento Grohmann Correio, continua as discussões sobre questões sonoras/auditivas, em especial sobre os estudos de música no Brasil. Os autores se concentram na metapesquisa de artigos do Encontro Nacional da Compós (2015 a 2019) e do Congresso Brasileiro de Ciências da Comunicação (2012 a 2019).

Já "Amorteamo", locus horribilis e a tropicalização do gótico na TV brasileira", de Anderson Lopes da Silva, aborda o elemento gótico nas produções culturais-midiáticas brasileiras, mais especificamente na ficção seriada televisiva. É o caso, por exemplo, da minissérie Amorteamo, exibida pela TV Globo. Thaís Costa da Silva, Maria Isabel Mendes de Almeida e Lilian Alves Gomes publicam aqui 
"Booktubers: Elogio da Materialidade e do Compartilhamento", texto que conecta reflexões de Walter Benjamin com os Booktubers, usuários de Internet que se dedicam apresentar vídeos com considerações sobre exemplares literários na Internet, com ênfase no YouTube. Ainda no campo da imagem, Fernão Ramos assina "Como é que é 'ver através' uma fotografia", estudo que conecta a Teoria da Fotografia com formulações filosóficas, como a perspectiva conceitual do pensamento analítico-cognitivo, em especial o trabalho de Kendall Walton. Para finalizar, o último texto da Seção Perspectiva, "Retornem para os seus países" parlamentares de cor : Trump, racismo e a repercussão na mídia negra dos EUA, de Ivonete da Silva Lopes, Gladys Mitchell-Walthour, artigo que analisa, sob a perspectiva da interseccionalidade, a repercussão das mensagens racistas do presidente Donald Trump na mídia negra dos Estados Unidos, em particular no episódio envolvendo as quatro parlamentares de cor estreantes no Congresso Nacional em 2016 pelo Partido Democrata.

A Eco-Pós publica ainda uma entrevista inédita com o cineasta Jonas Mekas, falecido em Janeiro de 2019. A conversa se deu em 2011 entre o cineasta e o pesquisador Vitor Zan, que na época realizava uma pesquisa sobre a obra do artista.

Terminamos 2020 com a publicação de três números bastante abrangentes e diversificados: "A Música e suas Determinações Materiais"; "O Pensamento Ecológico" e "Crise, feminismos e comunicação". Agradecemos a todxs que participaram e contribuíram para a existência da Revista ao longo desse ano tão conturbado. Voltaremos às atividades no primeiro semestre de 2021.

Desejamos uma boa leitura!

Liv Sovik (UFRJ)

Lígia Lana (PUC-RIO)

Maria Bogado (UFRJ)

Lucas Murari (UFRJ)

Com a colaboração da Equipe Editorial da Revista ECO-Pós. 


\section{EXPEDIENTE}

\section{EDITORAS ADJUNTAS}

Beatriz Jaguaribe, Universidade Federal do Rio de Janeiro, Brasil

Liv Sovik, Universidade Federal do Rio de Janeiro, Brasil

\section{EDITOR EXECUTIVO}

Lucas Murari, Universidade Federal do Rio de Janeiro, Brasil

EDITORA CONVIDADA

Lígia Lana, Pontifícia Universidade Católica do Rio de Janeiro, Brasil

\section{EDITORES ASSISTENTES}

Alexandre Kenichi Gouin, Universidade Federal do Rio de Janeiro, Brasil Luíza Alvim, Universidade Federal do Rio de Janeiro, Brasil Maria Bogado, Universidade Federal do Estado do Rio de Janeiro, Brasil Nicholas de Andueza Sineiro, Universidade Federal do Rio de Janeiro, Brasil Phillippe Sendas de Paula Fernandes, Universidade Federal do Rio de Janeiro, Brasil Vinícius Ferreira, Universidade Federal do Rio de Janeiro, Brasil

\section{COORDENADOR DE REVISÃO}

Augusto Flamaryon Cecchin Bozz, Universidade Federal do Rio de Janeiro, Brasil

\section{REVISÃO}

Ana Luiza Silva de Castro, Universidade Federal do Rio de Janeiro, Brasil Emily Almeida, Universidade Federal do Rio de Janeiro, Brasil Heitor Machado, Universidade Federal do Rio de Janeiro, Brasil Laianny Martins, Universidade Federal do Rio de Janeiro, Brasil Leonardo Motta, Universidade Federal do Rio de Janeiro, Brasil Liana Salles Monteiro, Universidade Federal do Rio de Janeiro, Brasil Ribamar Oliveira, Universidade Federal do Rio de Janeiro, Brasil Victor Terra, Universidade Federal do Rio de Janeiro, Brasil

\section{DIAGRAMAÇ̃̃O}

Ligia Barreto, Universidade Federal do Rio de Janeiro, Brasil Lucas Murari, Universidade Federal do Rio de Janeiro, Brasil 


\section{CONSELHO EDITORIAL}

Ana Paula Goulart Ribeiro, Universidade Federal do Rio de Janeiro, Brasil Andrew Calabrese, Universityof Colorado, Estados Unidos Antônio Fausto Neto, Universidade do Vale do Rio dos Sinos, Brasil Ben Singer, University of Wisconsin, Estados Unidos Bruno Campanella, Universidade Federal Fluminense, Brasil Dênis de Moraes, Universidade Federal Fluminense, Brasil Erick Felinto, Universidade do Estado do Rio de Janeiro, Brasil Francisco Rüdiger, PUC-RS, Brasil Guillermo Mastrini, Universidade de Buenos Aires, Argentina Gunhild Agger, Universidade de Aalborg, Dinamarca Horace Newcomb, Georgia University, Estados Unidos Itania Gomes, Universidade Federal da Bahia, Brasil Kátia Lerner, Fundação Oswaldo Cruz, Brasil Luis Albornoz, Universidad Carlos III de Madrid, Espanha Luis Felipe Miguel, Universidade de Brasília, Brasil Lynn Spigel, Northwestern University, Estados Unidos Márcia Benetti, Universidade Federal do Rio Grande do Sul, Brasil Marco Antonio Roxo da Silva, Universidade Federal Fluminense, Brasil Maria Helena Weber, Universidade Federal do Rio Grande do Sul, Brasil Maria Immacolata Vassalo Lopes, Universidade de São Paulo, Brasil Mateus Araújo, Universidade de São Paulo, Brasil Michael Schudson, Columbia University, Estados Unidos Mirta Varela, Universidade de Buenos Aires, Argentina Muniz Sodré, Universidade Federal do Rio de Janeiro, Brasil Othon Jambeiro, Universidade Federal da Bahia, Brasil Vanessa R. Schwartz, Universidade de Princeton, Estados Unidos Vera França, Universidade Federal de Minas Gerais, Brasil

\section{PARECERISTAS DA EDICÃ̃O}

Adriana Azevedo , Pontifícia Universidade Católica do Rio de Janeiro, Brasil Adriana da Rosa Amaral, Universidade do Vale do Rio dos Sinos, Brasil Agda Regina de Carvalho, Universidade Anhembi Morumbi, Brasil Aline Corso, Universidade do Vale do Rio dos Sinos, Brasil Amanda Medeiros, Universidade Federal do Rio de Janeiro, Brasil Ana Enne, Universidade Federal Fluminense, Brasil. Ana Maria da Conceição Veloso, Universidade Federal de Pernambuco, Brasil Anelise Angeli De Carli, Universidade Federal do Rio Grande do Sul, Brasil Beatriz Beraldo Batista, Centro Universitário Hermínio da Silveira, Brasil Beatriz Jaguaribe, Universidade Federal do Rio de Janeiro, Brasil.

Bruna Sant Ana Aucar, Pontifícia Universidade Católica do Rio de Janeiro, Brasil Camila Bastos Bacellar, Universidade Federal Fluminense, Brasil. 
Cíntia Guedes, Universidade Federal da Bahia, Brasil.

Claudia Junqueira de Lima Costa, Universidade Federal de Santa Catarina, Brasil

Claudia Mesquita, Universidade Federal de Minas Gerais, Brasil.

Cynthia Mara Miranda, Universidade Federal do Tocantins, Brasil

Daniel Serravalle de Sá, Universidade Federal de Santa Catarina, Brasil

Dantielli Assumpção Garcia, Universidade Estadual do Oeste do Paraná, Brasil

Danilo Postinguel, Centro Universitário UNIFIAM-FAAM, Brasil

Dayana Karla Melo da Silva, Universidade de São Paulo, Brasil

Érico Araújo Lima, Universidade Federal do Ceará, Brasil

Eliane Hatherly Paz, Universidade Federal do Rio de Janeiro, Brasil

Fernanda Bruno, Universidade Federal do Rio de Janeiro, Brasil

Fernanda Nascimento, Universidade Federal de Santa Catarina, Brasil

Fernando Gonçalves, Universidade Estadual do Rio de Janeiro, Brasil

Fernando Pocahy, Universidade do Estado do Rio de Janeiro , Brasil

Flávia Gabriela da Costa Rosa, Centro Universitário Teresa D`Ávila, Brasil

Francisco José Paoliello Pimenta, Universidade Federal de Juiz de Fora, Brasil

Gilberto Sobrinho, Universidade Estadual de Campinas, Brasil

Giselle Gomes da Silva Prazeres Souza, Universidade Federal Rural de Pernambuco, Brasil

Graciela Natansohn, Universidad Autónoma de México, México

Guiomar Ramos, Universidade Federal do Rio de Janeiro, Brasil

Iara Aparecida Beleli, Universidade Estadual de Campinas, Brasil

João Lucas Faco Tziminadis, Universität Erfurt, Alemanha

Josefina de Fatima Tranquilin-Silva, Universidade de Sorocaba, Brasil

Joyce Amâncio de Aquino Alves, Universidade da Integração Internacional da Lusofonia

Afro-Brasileira, Brasil

Karla Holanda, Universidade Federal Fluminense, Brasil

Kênia Freitas, Universidade Estadual Paulista Júlio de Mesquita Filho, Brasil

Lorena Christina Barros Travassos, Universidade Nova de Lisboa, Portugal

Luiza Helena Guimarães, Pontifícia Universidade Católica de São Paulo, Brasil

Maria Alice de Faria Nogueira, Universidade Federal do Rio de Janeiro, Brasil.

Maria da Graça Costa, Universidade Federal do Rio Grande do Norte, Brasil.

Maria Inês Amarante, Universidade Federal da Integração Latino Americana, Brasil

Mariana Baltar, Universidade Federal Fluminense , Brasil.

Marina Tedesco, Universidade Federal Fluminense, Brasil

Marlúcia Nogueira do Nascimento, Universidade Federal do Ceará, Brasil

Mateus Araújo Silva, Universidade de São Paulo, Brasil

Mayka Castellano, Universidade Federal Fluminense, Brasil

Michelly Carvalho, Universidade Federal do Maranhão, Brasil

Naara Lúcia de Albuquerque Luna, Universidade Federal Rural do Rio de Janeiro, Brasil

Nathan Menezes Amarante Teixeira, Universidade do Estado do Rio de Janeiro, Brasil

Pablo Nabarrete Bastos, Universidade Federal Fluminense, Brasil

Patricia Furtado M. Machado, Pontifícia Universidade Católica do Rio de Janeiro, Brasil

Patrícia Mourão, Universidade de São Paulo, Brasil

Paula Guimarães Simões, Universidade Federal de Minas Gerais, Brasil

Paula Izabela Nogueira Bartkiw, Universidade Tuiuti do Paraná, Brasil

Dossiê Crise, Feminismo e Comunicação - https://revistaecopos.eco.ufrj.br/

ISSN 2175-8689 - v. 23, n. 3, 2020

DOI: 10.29146/eco-pos.v23i3.27641 
Paulo Faltay, Universidade Federal do Rio de Janeiro, Brasil

Paulo Victor Barbosa de Sousa, Universidade Federal do Ceará, Brasil

Pedro Lapera, Universidade Federal Fluminense, Brasil.

Priscila Menezes, Universidade Federal do Estado do Rio de Janeiro, Brasil.

Rafael Teixeira, Pontifícia Universidade Católica do Rio de Janeiro, Brasil

Renata Rezende Ribeiro, Universidade Federal Fluminense, Brasil.

Rodolfo Rorato Londero, Universidade Estadual de Londrina, Brasil

Rodolfo Viana de Paulo, Universidade Federal Fluminense, Brasil

Rodrigo Sombra, Universidade Federal do Mato Grosso do Sul, Brasil

Sérgio Amadeu da Silveira, Universidade Federal do ABC, Brasil

Sheila Doula, Universidade Federal de Viçosa, Brasil

Simone Maria Rocha, Universidade Federal de Minas Gerais, Brasil

Sylvia Beatriz Bezerra Furtado, Universidade Federal do Ceará, Brasil

Tiago Salgado, Universidade Federal de Minas Gerais, Brasil 